\title{
A Study on the Effects of Plug Shape on Operating Performance of an Electric Pressure Regulator Applied for Gaseous Fueled Vehicles
}

\author{
Nguyen Ba Hung ${ }^{*}$, Le Anh Tuan', Ocktaeck Lim ${ }^{3}$ \\ ${ }^{1}$ School of Mechanical Engineering, Hanoi University of Science and Technology, Hanoi, Vietnam \\ ${ }^{2}$ School of Transportation Engineering, Hanoi University of Science and Technology, Hanoi, Vietnam \\ ${ }^{3}$ School of Mechanical Engineering, University of Ulsan, Ulsan, Republic of Korea \\ "Email: hung.nguyenba1@hust.edu.vn
}

\begin{abstract}
A model-based study was conducted to examine the effects of plug shape on electromagnetic force and dynamic response of an electric pressure regulator (EPR) applied for gaseous fueled vehicles. Mathematical models were established to describe the operation of the EPR, including mechanical and electrical models. A two-dimensional (2D) symmetric model of the EPR was built in Maxwell software to simulate the electromagnetic force under the effects of plug shape. Afterward, the $2 D$ symmetric model of EPR with the electromagnetic force calculated was imported into Simplorer software to simulate the dynamic response of the EPR based on the influence of plug shape. The shape of plug in the EPR was changed through the dimension parameters denoted by dimension $(h)$ and slope angle $(\alpha)$. The simulation results show that the electromagnetic force and dynamic response of the EPR can be optimized when $h$ and $\alpha$ are selected at $3 \mathrm{~mm}$ and $48^{\circ}$, respectively.
\end{abstract}

Keywords: Electric pressure regulator, plug shape, electromagnetic force, dynamic response

\section{Introduction}

The world is facing environmental pollution problems caused by exhaust gas from vehicles using gasoline and diesel fuels. Using natural gas fuel for vehicles is one of the ways to increase efficiency and reduce harmful emissions. In compressed natural gas $(\mathrm{CNG})$ vehicles, a fuel injection system integrated with a pressure regulator plays an important role in improving the performance and stability of the engine. A normal pressure regulator using a mechanical mechanism can limit the operating range of the control pressure due to its self-regulating properties. To increase the operating range of pressure regulators, an electromagnetic pressure regulator (EPR), or called solenoid pressure regulator (SPR), is utilized as an alternative solution. The EPR is an electromechanical device including a solenoid valve, utilized to continuously control the gas pressure providing to an injector of a gaseous fuel injection system. EPR's combination with the fuel injection system using gaseous fuels such as hydrogen and compressed natural gas $(\mathrm{CNG})$ is considered a potential solution to further improve engine efficiency and reduce emissions [1-4].

The operating performance of the EPR depends on electromagnetic force of the solenoid valve. The previous studies concentrated on improving the electromagnetic force of solenoids based examination of structural parameters [5-12]. Hung et al. [5] developed a solenoid applied for a gas injector, in which they varied structural parameters such as plunger mass, spring stiffness, and coil turns to increase the electromagnetic force. Yin and $\mathrm{Wu}$ [6] used Matlab/Simulink to simulate the electromagnetic force, open and close characteristics of a solenoid valve in a gas injection system under the influence of coil turns and air gap. Liu et al. [7] utilized Maxwell software to simulate the electromagnetic force of a solenoid valve applied for an electronic control fuel system based on the effects of structural parameters. Their simulation results showed that six interaction factors, including working air gap with armature radius, drive current with armature thickness, coil turns with side pole radius, armature thickness with its radius, armature thickness with side pole radius, and armature radius with side pole radius had a significant influence on the electromagnetic force. Shin and his research group [8] applied Maxwell software to analyze the effects of design parameters on electromagnetic dynamics of a solenoid valve used for an automotive fuel pump.

Hwang et al. [9] investigated the effects of input parameters such as input voltage, wire diameters and winding numbers on the performance of a solenoid used for diesel injector. Their results showed that the response time of the solenoid depended more on the winding number of the wire than on the wire diameter. In a study conducted by De et al. [10], the increase of

ISSN: 2734-9373

https://doi.org/10.51316/jst.152.ssad.2021.31.2.11

Received: December 23, 2020; accepted: May 13, 2021 
coil turns in a solenoid valve led to greater electromagnetic force. Cvetkovic et al. [11] used a modeling approach to develop a high-performance small size solenoid applied for a fuel injector. They used permanent magnets made from different materials to add inside the solenoid. The effects of five types of magnets on the attraction force of the solenoid were investigated in their study, including Neo35, $\mathrm{NdFe} 35, \mathrm{NdFe} 30, \mathrm{SmCo} 24$, and $\mathrm{SmCo} 28$. In addition, they also investigated the attraction force under the effects of different plunger pole shapes. Their results showed that the initial size of the fuel injector could be reduced by $35 \%$, the attraction force increased by $26 \%$, and the response time reduced by $76 \%$ by using the developed approach. Zhao et al. [12] tested the effects of drive current and the air gap between the armature and iron core on the electromagnetic force of a highspeed solenoid valve in a common rail injector based on electromagnetic models. Their simulation results showed that when the air gap decreased, the electromagnetic force increased. Besides the studies mentioned above, there are also other studies related to improving the operating performance of solenoids under influence of working conditions and structural parameters [13-16].

Among the structural parameters, the plug shape is considered one of the key parameters affecting the electromagnetic force as well as operating performance of a solenoid valve, which is rarely mentioned in the previous studies.

This paper presents a study on the influence of the plug shape on the electromagnetic force and dynamic response of an EPR applied for vehicles fueled with gaseous fuel or CNG vehicles. Mathematical models are established first to describe the operation of the EPR. A two-direction (2D) symmetric model of the EPR is built-in Maxwell software to simulate the electromagnetic force based on the influence of plug shape. Then the $2 \mathrm{D}$ model with the electromagnetic force simulated is imported into a Simplorer software to simulate the dynamic response of the EPR. The EPR model in Simplorer is created based on mathematical models established previously.

\section{Simulation Models}

\subsection{Mechanical Model}

In order to describe the mechanical operation of an EPR, an operating model and a force analysis model are shown in Fig. 1a,b, respectively. When the EPR is activated by input current, the plunger is moved in upward under the support of electromagnetic force and gas force. However, its motion is also affected by resistance forces such as gravitational force and elastic force caused by plunger mass and spring, respectively. Table 1 shows the specifications of an EPR, in which the EPR is designed with a range of inlet maximum gas pressure from 20 bar to 30 bar. The plunger motion obeys the second law of Newton, as described by the following equations:

$$
\begin{aligned}
& F_{e}-F_{s}-F_{d}+F_{p}-F_{g}=m \frac{d^{2} x}{d t^{2}} \\
& F_{e}-k(x+\Delta)-b \frac{d x}{d t}+P_{i n} S_{o}-m g=m \frac{d^{2} x}{d t^{2}}
\end{aligned}
$$

where, $F_{e}$ is electromagnetic force, $F_{s}$ is spring force, $F_{d}$ is damping force, $F_{g}$ is gravitational force, $F_{p}$ is pressure force, $k$ is spring stiffness, $\Delta$ is initial compression, $m$ is plunger mass, $P_{i n}$ is inlet pressure, $S_{o}$ is orifice cross-sectional area, and $x$ is plunger displacement of the EPR.

\subsection{Electrical Model}

Electromagnetic force appears when the coil of an EPR is provided by input voltage, which helps the plunger to move in upward, as shown in Fig. 1b.

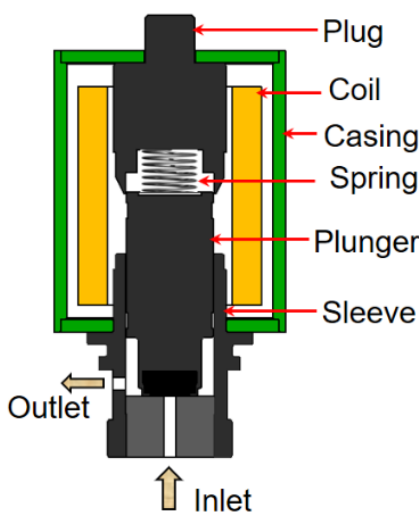

(a)

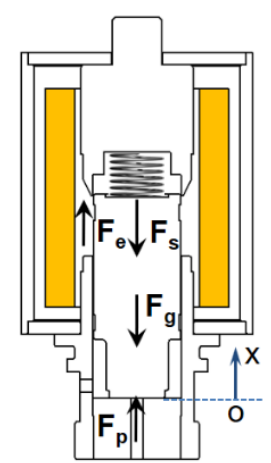

(b)
Fig. 1. Electric pressure regulator (EPR) with (a) operating model and (b) force analysis model.

The input voltage is described as shown below [2]:

$$
v_{0}=R i+\frac{d \lambda}{d t}
$$

where $\lambda$ is the total flux in the EPR, $v_{o}$ is the voltage providing to the EPR, $i$ is the current in the coil of the EPR, $R$ is the coil resistance of the EPR. $\lambda$ is also described as a function of current:

$$
\lambda=L(x) i
$$

where $L(x)$ is the inductance of the EPR. 
The voltage and current providing to the coil of the EPR are derived by combining (3) and (4):

$$
\begin{aligned}
& v_{0}=R i+L(x) \frac{d i}{d t}+i \frac{d L(x)}{d x} \frac{d x}{d t} \\
& \frac{d i}{d t}=\frac{1}{L(x)}\left[v_{0}-R i-i \frac{d L(x)}{d x} \frac{d x}{d t}\right]
\end{aligned}
$$

The current in Equation (6) is used as a variable to calculate the electromagnetic force which is presented by the following equations [17]:

$$
\begin{gathered}
W_{m}^{\prime}(i, x)=\int_{0}^{i_{r}} \lambda(i, x) d i=\int_{0}^{i_{r}} L(x) \cdot i d i=\frac{1}{2} i^{2} \cdot L(x) \\
F_{e}=\frac{\partial W_{m}^{\prime}(i, x)}{\partial x}=\frac{1}{2} i^{2} \cdot \frac{d L(x)}{d x}
\end{gathered}
$$

where, $W_{m}^{\prime}(i, x)$ is co-energy [17], which is a function of the inductance and current in the coil

The inductance of the EPR is defined by [18]:

$$
L(x)=\frac{N^{2}}{\sum_{1}^{n} \mathfrak{R}_{i}}
$$

where $\sum_{1}^{n} \mathfrak{R}_{i}$ is the total reluctance of the EPR and $\mathrm{N}_{\mathrm{r}}$ is coil turns number.

\subsection{EPR Model in Maxwell}

A 2D (two dimensional) drawing of the EPR created in computer-aided design (CAD) software is imported into Maxwell software to simulate the electromagnetic force based on operating and structure parameters of a real EPR. To reduce computation cost, a 2D model of the EPR in Maxwell is created with a symmetric type as presented in Fig. 2a, in which plunger, sleeve and plug are assigned with the stainless steel material S416, while the materials of the coil and casing are copper and stainless steel S430, respectively.

\subsection{Model of EPR in Simplorer}

A model of the EPR is built-in Simplorer software to simulate dynamic characteristics, which is based on mechanical and electrical models presented above. Therein, this model utilizes the EPR model in Maxwell as an input parameter. Therefore, the dynamic characteristics of EPR are simulated based on the influence of the plug shape. The model of EPR in Simplorer is presented in Fig. 3, in which the initial parameters of this model are the EPR model in Maxwell and specifications shown in Table 1.

Table 1. Specifications of EPR

\begin{tabular}{lc}
\hline \multicolumn{1}{c}{ Parameters } & Value \\
\hline Input current $(\mathrm{A})$ & 2.9 \\
Resistance of the coil $(\Omega)$ & 4.1 \\
Coil turns number & 600 \\
Mass of plunger $(\mathrm{g})$ & 31 \\
Spring hardness $(\mathrm{N} / \mathrm{m})$ & 2371 \\
\hline
\end{tabular}

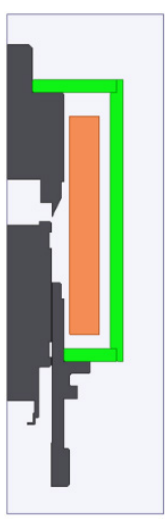

(a)

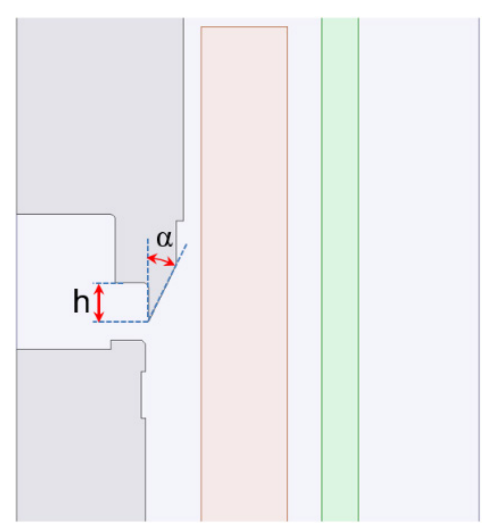

(b)
Fig. 2. EPR with (a) 2D symmetric model in Maxwell, and (b) dimension parameters of plug

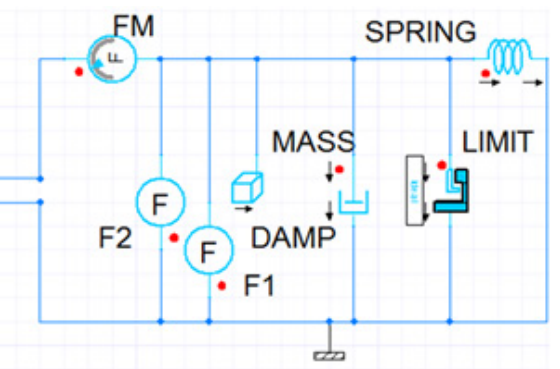

Fig. 3. Model of EPR in Simplorer. 


\section{Results and Discussions}

\subsection{Effects of Plug Shape on Electromagnetic Force}

Plug shape is changed through the variation of dimension $h$ and slope angle $\alpha$ as described in Fig. 2b, which relates to magnetic flux line toward the plunger, formation of the magnetic field strength in the EPR, as well as electromagnetic force acting on the plunger. Effects of $h$ on the electromagnetic force are depicted in Fig. 4, in which $h$ is varied at $2 \mathrm{~mm}, 3 \mathrm{~mm}$, and $4 \mathrm{~mm}$. In the initial position of the plunger $(x=0 \mathrm{~mm})$, the simulation results show that the electromagnetic force is smallest $(35.2 \mathrm{~N})$ when $h$ is varied at $2 \mathrm{~mm}$. Conversely, when $h$ is increased to $3 \mathrm{~mm}$, the electromagnetic force obtains the largest value $(44.6 \mathrm{~N})$ due to the closest distance created between the plunger head and plug bottom. The electromagnetic force is then reduced as $h$ is increased to $4 \mathrm{~mm}$. In the case of $h=4 \mathrm{~mm}$, the plunger head tends to leave the plug bottom as the plunger stroke is increased from $0 \mathrm{~mm}$ to $2 \mathrm{~mm}$, thus the electromagnetic force has a reduced trend, as observed in Fig. 4. On the contrary, the electromagnetic force has an increasing trend for the case of $h=2 \mathrm{~mm}$ because the plunger head tends to reach closer to the plug bottom. For the case of $h=3 \mathrm{~mm}$, the electromagnetic force tends to remain nearly unchanged value during the plunger stroke from $0 \mathrm{~mm}$ to $2 \mathrm{~mm}$. When the plunger stroke is increased from $2 \mathrm{~mm}$ to $3 \mathrm{~mm}$, the electromagnetic force increases accordingly for all three cases of changing $h$. This can be explained by the increased magnetic density as the air gap between the plunger and plug is reduced [6].

As can be seen in Fig. 5, the electromagnetic force increases significantly at plunger stroke $x=0 \mathrm{~mm}$

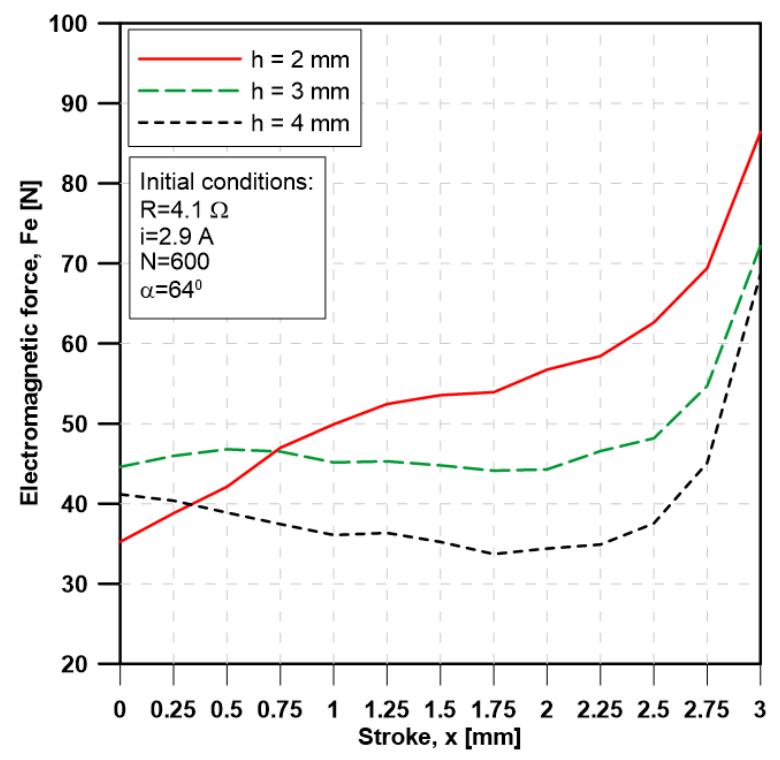

Fig. 4. Effects of dimension $h$ on the electromagnetic force as $\alpha$ is adjusted from $0^{0}$ to $48^{0}$. When $\alpha$ is continued to increase to $64^{\circ}$ and $80^{\circ}$, the electromagnetic force has a reduced tendency at plunger stroke $x=0 \mathrm{~mm}$, which can be explained by the reduced magnetic flux line due to the increase of slope angle $\alpha$. However, when the plunger stroke is increased, an opposite trend is observed for the variation of electromagnetic force via $\alpha$. By increasing $\alpha$ from $0^{0}$ to $48^{0}$, the electromagnetic force has a reduced tendency during the plunger stroke from $0 \mathrm{~mm}$ to $2 \mathrm{~mm}$. When $\alpha$ is increased to $64^{\circ}$ and $80^{\circ}$, the electromagnetic force tends to increase as the plunger stroke is adjusted from $0 \mathrm{~mm}$ to $2 \mathrm{~mm}$. The better oriented magnetic flux line created by increasing the slope angle $\alpha$ can be considered as one cause of this phenomenon.

The simulation results in Fig. 5 show that the electromagnetic force is increased for all cases of changing $\alpha$ as the plunger stroke is increased from 2 $\mathrm{mm}$ to $3 \mathrm{~mm}$, which has a similar trend when compared with the simulation results in Fig. 4. Fig. 6 presents the effects of slope angle $\alpha$ on the magnetic flux line and field strength, in which $\alpha$ is varied at $48^{\circ}, 64^{\circ}$ and $80^{\circ}$. It can be seen that the increase of $\alpha$ results in the decrease of magnetic flux line and field strength, which leads to decreasing the electromagnetic force at the initial position of the plunger as described in Fig. 5.

Fig. 5 shows the effects of slope angle $\alpha$ on the electromagnetic force versus plunger stroke, in which the value of $\alpha$ is increased from $0^{0}$ to $80^{\circ}$ with an increment of $16^{0}$.

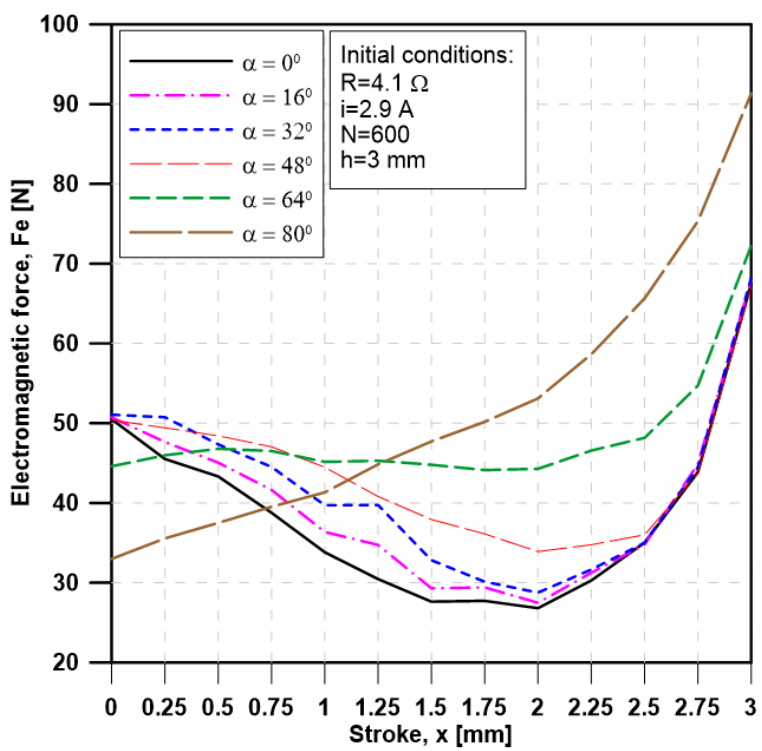

Fig. 5. Effects of slope angle $\alpha$ on the electromagnetic force 


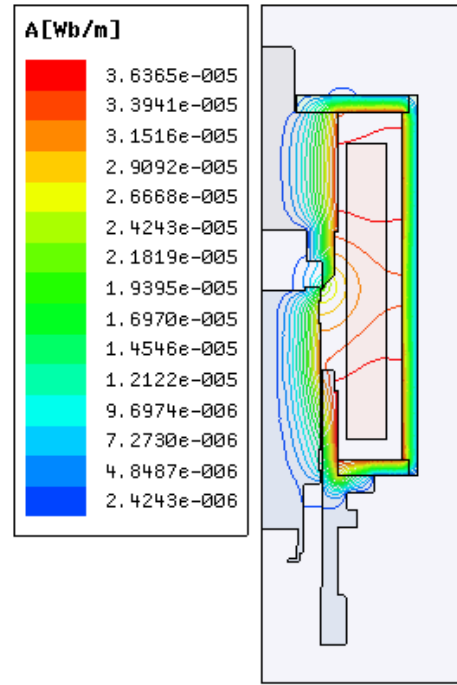

(a)

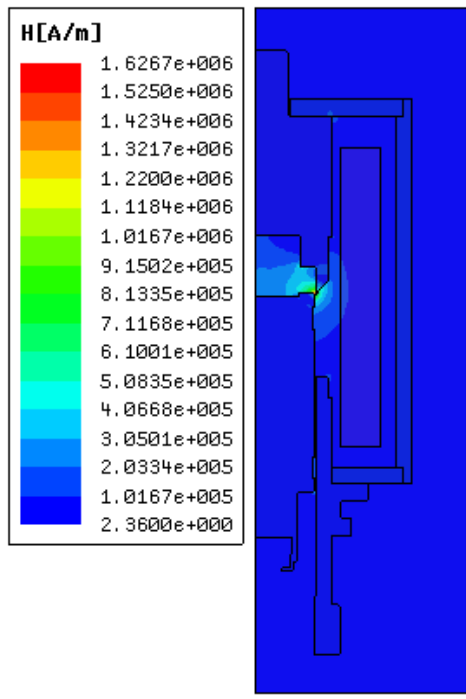

(d)

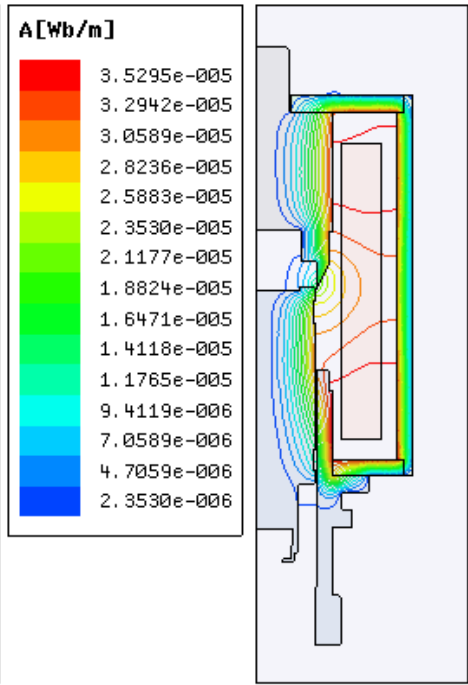

(b)

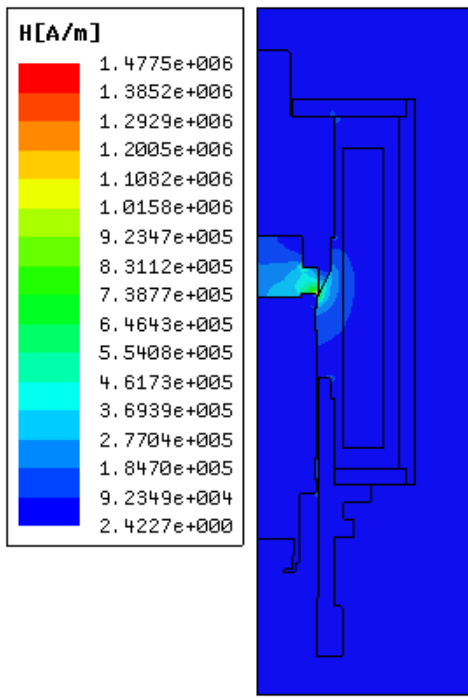

(e)

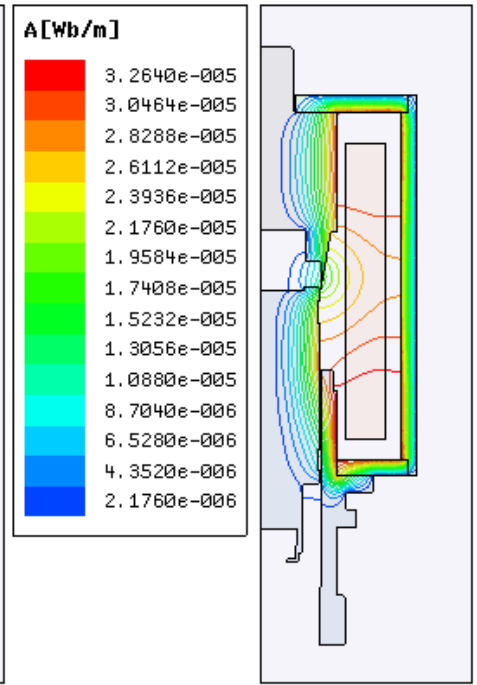

(c)
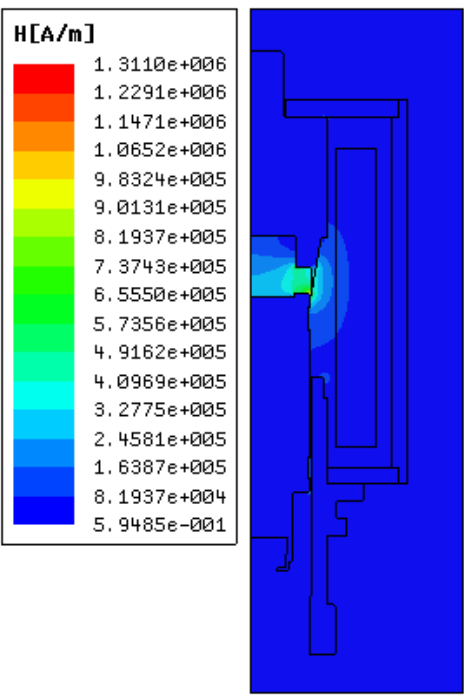

(f)

Fig. 6. Magnetic flux lines $(A)$ and field strength $(H)$ with $(a),(d) \alpha=48^{\circ} ;(b),(e) \alpha=64^{0}$ and $(c),(f) \alpha=80^{0}$

\subsection{Dynamic Response of EPR}

The effects of dimension $h$ on the electromagnetic force of the EPR in the transient mode are shown in Fig. 7. The simulation results show that the electromagnetic force increases quickly during $10 \mathrm{~ms}$ of open stroke, in which the increased speed of the electromagnetic force corresponding to $h=3 \mathrm{~mm}$ is the fastest. This can be explained by the rapid increase of plunger displacement as shown in Fig. 8.

When $h$ is increased to $4 \mathrm{~mm}$, a fluctuation of electromagnetic force is observed. This can be due to the influence of spring force as the electromagnetic force is reduced, as observed in Fig. 4. As the result, the fluctuation of electromagnetic force leads to the fluctuation of plunger displacement as observed in Fig. 8. When the input voltage is stopped providing to the coil at $50 \mathrm{~ms}$, the electromagnetic force is reduced, and the plunger tends to close the inlet port (close stroke). It can be seen that the close of inlet port take places sooner when $h$ is increased from $2 \mathrm{~mm}$ to $4 \mathrm{~mm}$, which is observed by the earlier reduction of plunger displacement, as shown in Fig. 8. This can be explained by the earlier reduction of the electromagnetic force as $\mathrm{h}$ is increased as observed in Fig. 7.

Fig. 9 depicts the effects of slope angle $\alpha$ on the electromagnetic force in the transient mode. The simulation results show that the electromagnetic force increases unstably in the open stroke when $\alpha$ is changed from $0^{0}$ to $48^{0}$. This can be due to the reduced trend of the electromagnetic force during the plunger stroke from $0 \mathrm{~mm}$ to $2 \mathrm{~mm}$, as observed in Fig. 5 . When $\alpha$ is increased to $64^{0}$, the fluctuation of electromagnetic force is reduced due to its stability as seen in Fig. 5. However, a big fluctuation of electromagnetic force in open stroke occurs as the slope angle $\alpha$ is increased to $80^{\circ}$. The small 
electromagnetic force in the initial position of plunger along with the effects of spring force can be causes of this big fluctuation. The big fluctuation of electromagnetic force results in the big fluctuation of plunger displacement in the open stroke, as shown in Fig. 10.

When the input voltage is stopped supplying to the coil at $50 \mathrm{~ms}$, the electromagnetic force tends to reduce, and the close process of the inlet port is taken

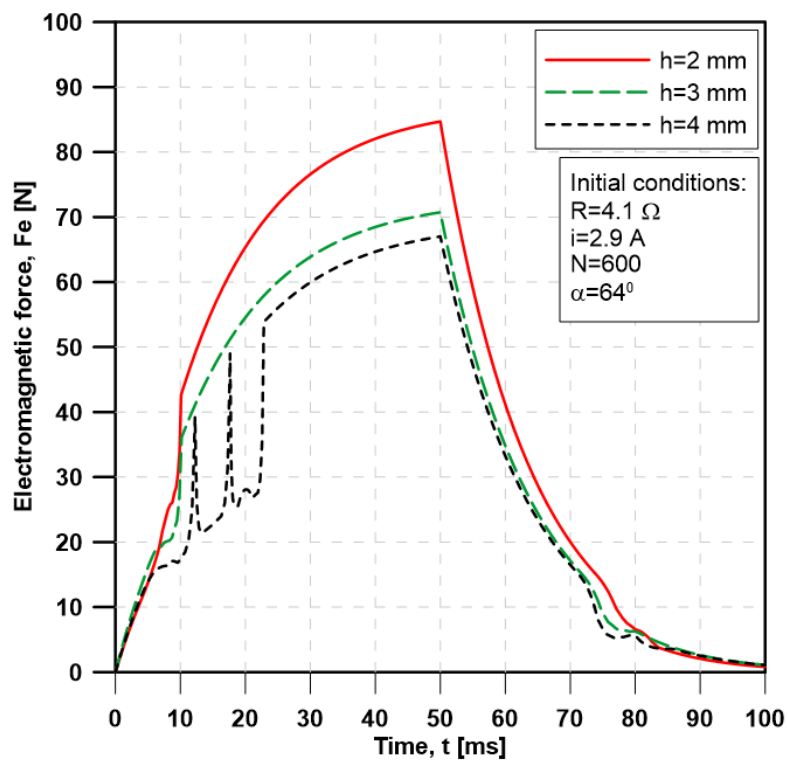

Fig. 7. Effects of dimension $h$ on the electromagnetic force in the transient mode

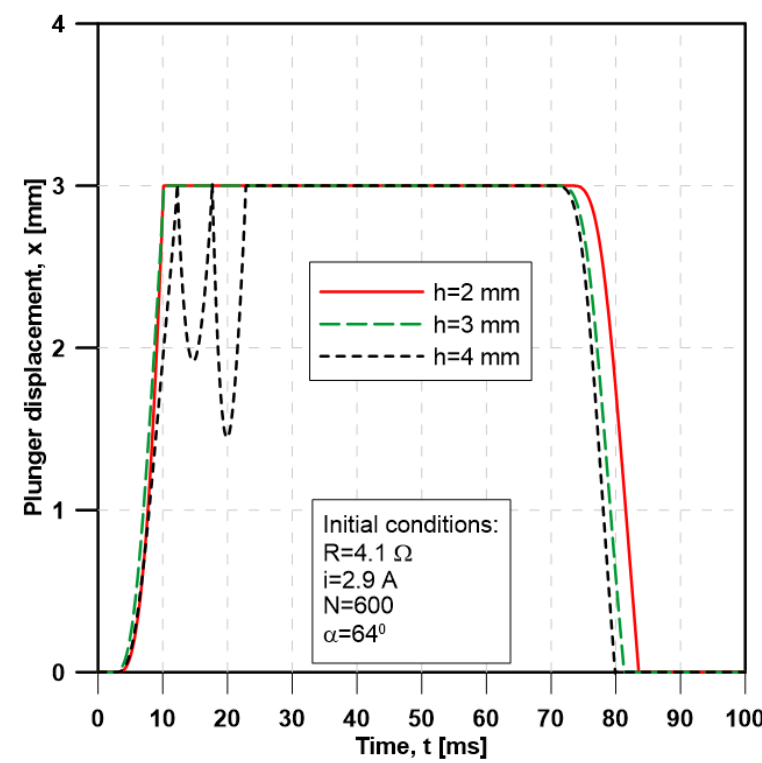

Fig. 8. Effects of dimension $h$ on the plunger displacement place as the electromagnetic force is smaller than the resistance forces caused by spring and gas pressure. As can be seen in Fig. 10, the plunger displacement is reduced earlier when the slope angle $\alpha$ is changed from $0^{0}$ to $48^{0}$, when compared with the reduction of electromagnetic force corresponding to $\alpha=64$ and $\alpha=80^{\circ}$. This is due to the influence of electromagnetic force as described in Fig. 9.

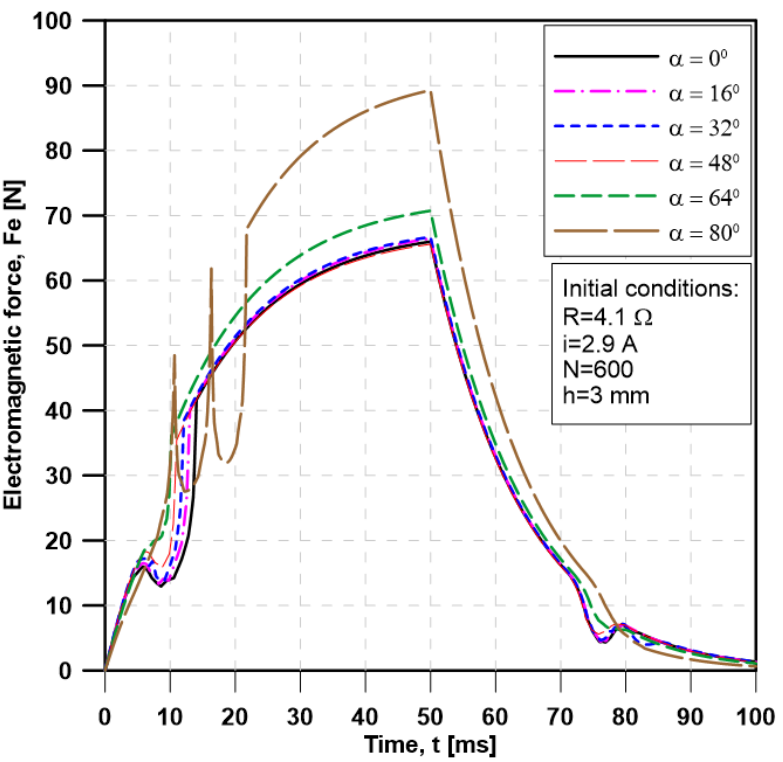

Fig. 9. Effects of slope angle $\alpha$ on the electromagnetic force in the transient mode.

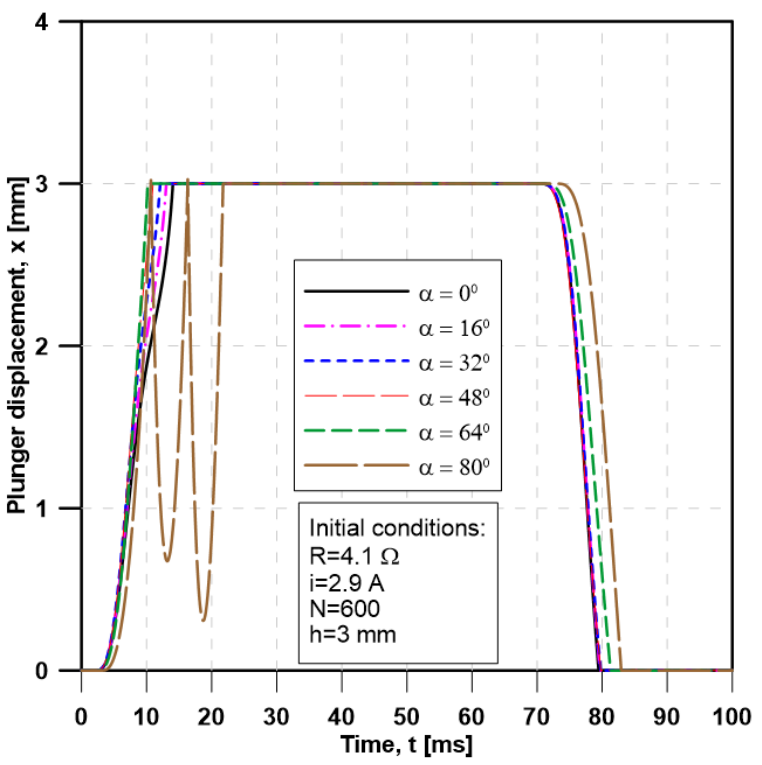

Fig. 10. Effects of slope angle $\alpha$ on the plunger displacement. 


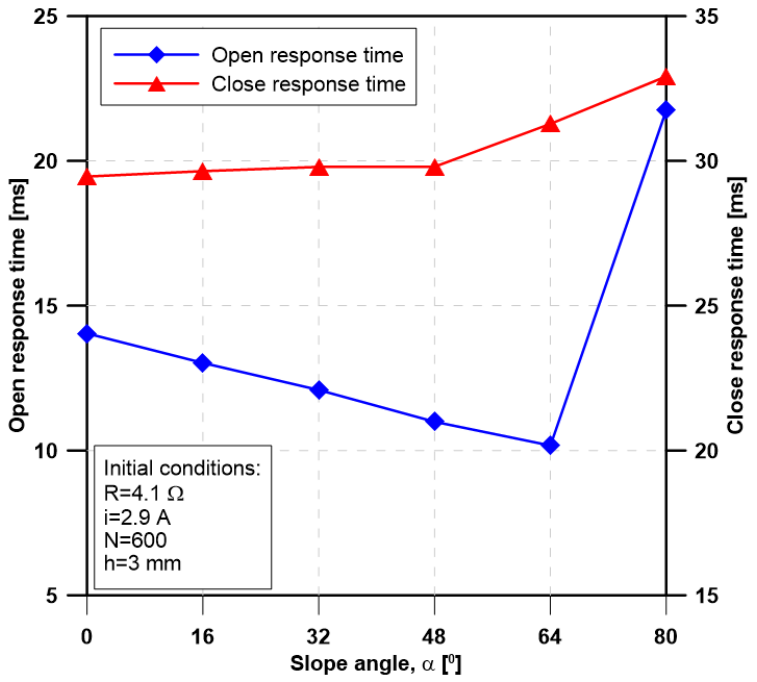

Fig. 11. Open and close response time under the effects of slope angle $\alpha$.

Fig. 11 shows the open and close response time of the plunger based on the simulation results obtained in Fig. 10. It can be seen that the open response time is reduced when the slope angle $\alpha$ is changed from $0^{0}$ to $64^{\circ}$, and then increased significantly as $\alpha$ is increased to $80^{\circ}$ due to the fluctuation of plunger displacement. The close response time has an increasing trend when $\alpha$ is changed from $0^{0}$ to $80^{\circ}$, especially the increment is considerable as $\alpha$ is changed from $64^{\circ}$ to $80^{\circ}$. Therefore, $\alpha$ should be selected at $48^{\circ}$ as an optimum slope angle.

\section{Conclusion}

A model-based study to examine the effects of plug shape on the electromagnetic force and dynamic response of an EPR was conducted. The electromagnetic force and dynamic response of the EPR were simulated based on a combination of computer programs included Maxwell and Simplorer. The plug was changed in shape through the variation of its design parameters, such as dimension $h$ and slope angle $\alpha$.

The simulation results indicated that the variation of dimension $h$ and slope angle $\alpha$ had significant effects on the electromagnetic force as well as dynamic response of the EPR. A high-performance EPR could be obtained when the optimum values of $h$ and $\alpha$ were selected at $3 \mathrm{~mm}$ and $48^{\circ}$, respectively.

This study could be a useful reference for designing high-performance solenoids applied on gaseous fueled vehicles to increase efficiency and reduce exhaust emissions.

\section{Acknowledgments}

This research is funded by Vietnam National Foundation for Science and Technology Development (NAFOSTED) under grant number 107.01-2020.17

\section{References}

[1]. L. Ding, J. Wu, Innovation ecosystem of CNG vehicles: A case study of its cultivation and characteristics in Sichuan, China, Sustainability, vol. 10, pp. 1-16, 2018. https://doi.org/10.3390/su10010039

[2]. X. Wang, H, Zhang, B. Yao, Y. Lei, X. Sun, D. Wang, Y. Ge, Experimental study on factors affecting lean combustion limit of S.I engine fueled with compressed natural gas and hydrogen blends, Energy, vol. 38, pp. 58-65, 2012. https://doi.org/10.1016/j.energy.2011.12.042

[3]. J.A. Yamin, M.A. Hamdan, The performance of hydrogen-powered 4-stroke SI engine using locally designed fuel regulator, J. Braz. Soc. Mech. Sci. Eng., vol. 32, pp. 195-199, 2010. https://doi.org/10.1590/S1678-58782010000300001

[4]. M. Ehsan, Effect of spark advance on a gas run automotive spark ignition engine, J. Chem. Eng., vol. 24, pp. 42-49, 2006. https://doi.org/10.3329/jce.v24i0.5584

[5]. N.B. Hung, O.T. Lim, S.J. Yoon, Effects of structural parameters on operating characteristics of a solenoid injector, Energy Procedia, vol. 105, pp. 1771-1775, 2017.

https://doi.org/10.1016/j.egypro.2017.03.511

[6]. L. Yin, C. Wu, The characteristic analysis of the electromagnetic valve in opening and closing process for the gas injection system, J. Electromagn. Anal. Appl. vol. 8, pp. 152-159, 2016. https://doi.org/10.4236/jemaa.2016.88015

[7]. P. Liu, L. Fan, Q. Hayat, D. Xu, X. Ma, E. Song, Research on key factors and their interaction effects of electromagnetic force of high-speed solenoid valve, Sci. World J., vol. 2014, pp. 1-13, 2014. https://doi.org/10.1155/2014/567242

[8]. Y. Shin, S. Lee, C. Choi, J. Kim, Shape optimization to minimize the response time of direct-acting solenoid valve, J. Magn., vol. 20, pp. 193-200, 2015. https://doi.org/10.4283/JMAG.2015.20.2.193

[9]. J.W. Hwang, H.J. Kal, J.K. Park, A.A. Martychenko, and J.O. Chae, A study on the design and application of optimized solenoid for diesel unit injector. KSME International Journal, vol. 13, pp. 414-420, 1999. https://doi.org/10.1007/BF02939329

[10].X. De, F. Hong-Zi, L. Peng, Z. Wei, and F.L. Yun, Electromagnetic force on high-speed solenoid valve based on correlation analysis, International Journal on Smart Sensing and Intelligent Systems, vol. 8, pp. 22672285, 2015. https://doi.org/10.21307/ijssis-2017-853

[11].D. Cvetkovic, I. Cosic, and A. Subic, Improved performance of the electromagnetic fuel injector solenoid actuator using a modeling approach, International Journal of Applied Electromagnetics and Mechanics, vol. 27, pp. 251-273, 2008. https://doi.org/10.3233/JAE-2008-939

[12].J. Zhao, L. Fan, P. Liu, L. Grekhov, X. Ma, and E. Song, Investigation on electromagnetic models of high-speed 
solenoid valve for common rail injector, Mathematical Problems in Engineering, vol. 2017, pp. 1-10, 2017. https://doi.org/10.1155/2017/9078598

[13].A. Subic, and D. Cvetkovic, Virtual design and development of compact fast-acting fuel injector solenoid actuator, International Journal of Vehicle Design, vol. 46, pp. 309-327, 2008.

https://doi.org/10.1504/IJVD.2008.019089

[14].Q. Wang, F. Yang, Q. Yang, J. Chen, and H. Guan, Experimental analysis of new high-speed powerful digital solenoid valves, Energy Conversion and Management, vol. 52, pp. 2309-2313, 2011. https://doi.org/10.1016/j.enconman.2010.12.032

[15].L. Grekhov, J. Zhao, and X.Ma, Fast-Response solenoid actuator computational dimulation for engine fuel systems, International Conference on Industrial
Engineering, Applications and Manufacturing, Russia, 2017.

https://doi.org/10.1109/ICIEAM.2017.8076413

[16].N.B. Hung and O.T. Lim, A simulation and experimental study on the operating characteristics of a solenoid gas injector, Advances in Mechanical Engineering, vol. 11, pp. 1-14, 2019. https://doi.org/10.1177/1687814018817421

[17].H.H. Woodson, J.R. Melcher, Electromechanical Dynamics, John Wiley \& Sons Inc., New York, NY, USA, 1968.

[18].V. Giurgiutiu, S.E. Lysheyski, Micromechatronics: Modeling, Analysis, and Design with MATLAB, 2nd ed., CRC Press: Boca Raton, FL, USA, 2009. 